\title{
THE DIRICHLET PROBLEM FOR HOMOGENEOUS ELLIPTIC OPERATORS IN A HALF SPACE
}

BY J. BARROS-NETO

Communicated by F. Browder, June 22, 1964

In a recent series of papers, Lions and Magenes [4], [5] study extensively boundary-value problems for elliptic operators. A prototype of problem that cannot be attacked by their methods is the Dirichlet problem for powers of the Laplacian in a half space. By using the completion of the space of smooth functions with respect to the Dirichlet norm $(\$ 1)$, we are able to solve this problem.

We obtain, then, for a general class of homogeneous elliptic operators defined in a half space, isomorphism theorems establishing existence and uniqueness for the Dirichlet problem ( $\$ \S 2,4$ and 5), a regularity result $(\$ 3)$ and trace theorems ( $\$ 2,3$ and 4$)$. Using the theory of interpolation [3] we obtain other isomorphism theorems between general classes of interpolated spaces. Among these, we can characterize the spaces of boundary values (Theorem 5.2).

Proofs will appear elsewhere. The writer is indebted to J.-L. Lions for suggestions and criticism.

1. Preliminaries. Let $R^{n}$ be the Euclidean space of $n$ dimensions, its elements being denoted by $x=\left(x_{1}, \cdots, x_{n}\right)$. We denote by $R_{+}^{n}$ (resp. $\bar{R}_{+}^{n}$ ) the set of elements $x=\left(x_{1}, \cdots, x_{n}\right) \in R^{n}$ such that $x_{n}>0$ (resp. $x_{n} \geqq 0$ ). If $p=\left(p_{1}, \cdots, p_{n}\right)$ is an $n$-tuple of integers $\geqq 0$, let $D^{p}=D_{1}^{p_{1}}, \cdots, D_{n}^{p_{n}}$, where $D_{j}=(1 / i)\left(\partial / \partial x_{j}\right), 1 \leqq j \leqq n$, and let $|p|=p_{1}+\cdots+p_{n}$ be the order of $D^{p}$. If $\Omega$ is an open subset of $R^{n}$, we denote by $C_{c}^{\infty}(\Omega)$ the space of infinitely differentiable functions with compact support in $\Omega$.

Definition 1.1. We denote by $D^{m}\left(R^{n}\right)$ the completion of $C_{c}^{\infty}\left(R^{n}\right)$, with respect to the following norm:

$$
\|\phi\|_{D^{m}\left(R^{n}\right)}=\left(\sum_{|p|=m}\left\|D^{p} \phi\right\|_{L^{2}\left(R^{n}\right)}^{2}\right)^{1 / 2} .
$$

Clearly, $D^{m}\left(R^{n}\right)$ is a Hilbert space. If $n>2 m$ (an assumption that we shall make throughout this paper), we have the Sobolev inequality [6]:

$$
\|\phi\|_{L^{q(m)}\left(R^{n}\right)} \leqq c\|\phi\|_{D^{m}\left(R^{n}\right)}, \quad \text { for all } \phi \in C_{c}^{\infty}\left(R^{n}\right)
$$

where 


$$
\frac{1}{q(m)}=\frac{1}{2}-\frac{m}{n},
$$

and $c$ is a constant independent of $\phi$. It follows that $D^{m}\left(R^{n}\right) \subset L^{q(m)}\left(R^{n}\right)$, the imbedding being continuous. In fact, we have a more precise characterization of the elements of $D^{m}\left(R^{n}\right)$.

Theorem 1.1. The space $D^{m}\left(R^{n}\right)$ can be identified in the algebraic and topological senses to the space:

$$
\left\{u \in L^{q(m)}\left(R^{n}\right): D^{p} u \in L^{q(m-j)}\left(R^{n}\right),|p|=j, 0 \leqq j \leqq m\right\},
$$

where

$$
\frac{1}{q(m-j)}=\frac{1}{2}-\frac{m-j}{n}, \quad 0 \leqq j \leqq m,
$$

equipped with the norm

$$
\sum_{|p|=j=0}^{m}\left\|D^{p} u\right\|_{L^{q(m-j)}\left(R^{n}\right)}
$$

In order to study the Dirichlet problem for homogeneous differential operators of order $2 m$ in the half space $R_{+}^{n}$, we introduce the following

Definition 1.2. We denote by $D^{m}\left(R_{+}^{n}\right)$ the space $D^{m}\left(R_{+}^{n}\right)=\left\{u \in L^{q(m)}\left(R_{+}^{n}\right): D^{p} u \in L^{q(m-j)}\left(R_{+}^{n}\right),|p|=j, 0 \leqq j \leqq m\right\}$, where $q(m-j)$ is given by (1.4), equipped with the norm

$$
\|u\|_{D^{m}\left(R_{+}^{n}\right)}=\sum_{|p|=j=0}^{m}\left\|D^{p} u\right\|_{L^{q(m-j)}\left(R_{+}^{n}\right)^{\circ}}
$$

Also let $D_{0}^{m}\left(R_{+}^{n}\right)$ be the closure of $C_{c}^{\infty}\left(R_{+}^{n}\right)$ in $D^{m}\left(R_{+}^{n}\right)$ and let $D^{-m}\left(R_{+}^{n}\right)$ be the dual of $D_{0}^{m}\left(R_{+}^{n}\right)$.

2. Isomorphism theorems. Consider now the integro-differential operator defined on $D^{m}\left(R_{+}^{n}\right) \times D^{m}\left(R_{+}^{n}\right)$,

$$
a(u, v)=\sum_{|p|=|q|=m} \int_{R_{+}^{n}} a_{p q}(x) D^{p} u \bar{D}^{a} v d x
$$

where we assume, for simplicity, that the $a_{p q}(x)$ are $C^{\infty}$ functions on $\bar{R}_{+}^{n}$ and uniformly bounded. Assume that $a(u, v)$ satisfies the ellipticity condition: 


$$
|a(v, v)| \geqq c\|v\|_{D^{m}\left(R_{+}^{n}\right)}^{2} \quad \text { for all } v \in D_{0}^{m}\left(R_{+}^{n}\right) .
$$

Then one can prove the following (Lax-Milgram lemma [2]).

THEOREM 2.1. Given an element $f \in D^{-m}\left(R_{+}^{n}\right)$ there is a unique $u \in D_{0}^{m}\left(R_{+}^{n}\right)$ such that

$$
a(u, v)=\langle f, v\rangle \quad \text { for all } v \in D_{0}^{m}\left(R_{+}^{n}\right),
$$

where $\langle$,$\rangle denotes the pairing between D_{0}^{m}\left(R_{+}^{n}\right)$ and $D^{-m}\left(R_{+}^{n}\right)$.

If

$$
A u=\sum_{|p|=|q|=m}(-1)^{m} D^{q}\left(a_{p q}(x) D^{p} u\right)
$$

is the differential operator associated to (2.1), we derive the

Corollary 2.1. $A$ is an isomorphism of $D_{0}^{m}\left(R_{+}^{n}\right)$ onto $D^{-m}\left(R_{+}^{n}\right)$.

In other words, the homogeneous Dirichlet problem for $A$ has a unique solution in $D_{0}^{m}\left(R_{+}^{n}\right)$ for any given element in $D^{-m}\left(R_{+}^{n}\right)$. In order to study the inhomogeneous Dirichlet problem (see (2.5)) we need to define the trace (i.e., restriction in a suitable sense) on $R^{n-1}$ of the functions of $D^{m}\left(R_{+}^{n}\right)$.

Definition 2.1. Let $s$ be a real number and denote by $D^{s}\left(R^{n-1}\right)$ the completion of $C_{c}^{\infty}\left(R^{n-1}\right)$ with respect to the norm

$$
\|\phi\|_{D^{s}\left(R^{n-1}\right)}=\left(\int_{R^{n-1}}\left|\xi^{\prime}\right|^{2 s}\left|\hat{\phi}\left(\xi^{\prime}\right)\right|^{2} d \xi^{\prime}\right)^{1 / 2}, \quad \phi \in C_{c}^{\infty}\left(R^{n-1}\right),
$$

where $\hat{\phi}$ denotes the Fourier transform of $\phi$ and $\xi^{\prime}=\left(\xi_{1}, \cdots, \xi_{n-1}\right)$.

One can see [1] that for $-(n-1) / 2<s<(n-1) / 2, D^{s}\left(R^{n-1}\right)$ is a subspace of $S^{\prime}\left(R^{n-1}\right)$, the space of temperated distributions in $R^{n-1}$; also the dual of $D^{s}\left(R^{n-1}\right)$ is $D^{-s}\left(R^{n-1}\right)$.

THEOREM 2.2. There is a continuous linear map

$$
\gamma=\left(\gamma_{0}, \cdots, \gamma_{m-1}\right): D^{m}\left(R_{+}^{n}\right) \rightarrow \prod_{j=0}^{m-1} D^{m-j-1 / 2}\left(R^{n-1}\right)
$$

with the following properties:

(i) for all functions $\phi$, infinitely differentiable and with compact support in $\bar{R}_{+}^{n}, \gamma_{j} \phi=\left(\partial^{j} \phi / \partial x_{n}^{j}\right)\left(x^{\prime}, 0\right)$, where $x^{\prime}$ denotes $\left(x_{1}, \cdots, x_{n-1}\right)$;

(ii) $\gamma$ is onto;

(iii) $\gamma^{-1}(0)=D_{0}^{m}\left(R_{+}^{n}\right)$. 
Using this theorem and Corollary 1.1 we easily derive the following result.

THEOREM 2.3. $(A, \gamma)$ is an isomorphism of $D^{m}\left(R_{+}^{n}\right)$ onto $D^{-m}\left(R_{+}^{n}\right)$ $\times \prod_{j=0}^{m-1} D^{m-j-1 / 2}\left(R^{n-1}\right)$.

In other words, the inhomogeneous Dirichlet problem

$$
\begin{aligned}
A u & =f, \\
\gamma_{j} u & =g_{j}, \quad 0 \leqq j \leqq m-1,
\end{aligned}
$$

has a unique solution in $D^{m}\left(R_{+}^{n}\right)$ for any given $f \in D^{-m}\left(R_{+}^{n}\right)$ and

$$
\left(g_{j}\right) \in \prod_{j=0}^{m-1} D^{m-j-1 / 2}\left(R^{n-1}\right) .
$$

3. Regularization. For any open subset $\Omega$ of $R^{n}$ let $W^{k, q}(\Omega)$ be the Sobolev space of functions $u \in L^{q}(\Omega)$ with derivatives $D^{p} u$, in the sense of distributions, belonging to $L^{q}(\Omega)$ for $|p| \leqq k$. When $q=2$, we denote $W^{m, 2}(\Omega)$ by $H^{m}(\Omega)$.

Definition 3.1. We denote by $D^{k, m}\left(R_{+}^{n}\right)$ ( $k$ integer $\geqq 0$ ) the space $D^{k, m}\left(R_{+}^{n}\right)=\left\{u \in W^{k, q(m)}\left(R_{+}^{n}\right): D^{p} u \in W^{k, q(m-j)}\left(R_{+}^{n}\right), 0 \leqq|p|=j \leqq m\right\}$, equipped with its natural norm.

Theorem 3.3. If $f \in D^{-(m-1)}\left(R_{+}^{n}\right) \cap D^{-m}\left(R_{+}^{n}\right)$, then the unique solution $u \in D_{0}^{m}\left(R_{+}^{n}\right)$ of (2.3) belongs to $D^{1, m}\left(R_{+}^{n}\right)$.

4. Transposition. Let $a^{*}(u, v)=(a(v, u))^{-}$and let $A^{*}$ be the formal adjoint of $A$. Suppose that $a^{*}(u, v)$ verifies condition $(2.2)$, thus all the previous results apply to $A^{*}$. In particular, Corollary 2.1 together with Theorem 3.3 hold.

TheOREM 4.1. $A^{*}$ is an isomorphism of $D^{m, m}\left(R_{+}^{n}\right) \cap D_{0}^{m}\left(R_{+}^{n}\right)$ onto $\bigcap_{p=0}^{m} D^{-p}\left(R_{+}^{n}\right)$. (Here $D^{0}\left(R_{+}^{n}\right)=L^{2}\left(R_{+}^{n}\right)$.)

If we transpose this result and use the same trick as in [4] we get

TheOREM 4.2. $(A, \gamma)$ is an isomorphism of $H$ onto

$$
D^{-m}\left(R_{+}^{n}\right) \times \prod_{j=0}^{m-1} H^{-j-1 / 2}\left(R^{n-1}\right),
$$

where $H$ is the space of functions w belonging to the dual of $\bigcap_{p=0}^{m} D^{-p}\left(R_{+}^{n}\right)$ such that $A w \in D^{-m}\left(R_{+}^{n}\right)$, equipped with its natural norm.

5. Interpolation. We can now obtain other isomorphism theorems by interpolating the results of Theorem 2.3 and of Theorem 4.2 . If 
$A$ and $B$ are two Banach spaces contained in a topological vector space $S$, if $1<p, q<+\infty, \alpha$ and $\beta$ two real numbers such that $1 / p+\alpha$ and $1 / q+\beta$ lie in the interval $(0,1)$, we shall consider the trace space $T(p, \alpha, A ; q, \beta, B)$, as introduced by Lions in [3]. This space verifies the interpolation property with respect to continuous linear maps [3, Theorem 3.1]. Applying this fact to our situation we get, taking $p=q=2, \alpha=\beta$, after an obvious change of notation:

Theorem 5.1. $(A, \gamma)$ is an isomorphism of $T\left(2, \alpha ; D^{m}\left(R_{+}^{n}\right), H\right)$ onto $D^{-m}\left(R_{+}^{n}\right) \times \prod_{j=0}^{m-1} T\left(2, \alpha ; D^{m-j-1 / 2}\left(R^{n-1}\right), H^{-(j+1 / 2)}\left(R^{n-1}\right)\right)$.

The significance of this result lies in the fact that we can characterize in an explicit way the elements of $T\left(2, \alpha ; D^{m-j-1 / 2}, H^{-(j+1 / 2)}\right)$.

Theorem 5.2. The space $T\left(2, \alpha ; D^{m-j-1 / 2}\left(R_{+}^{n}\right), H^{-(j+1 / 2)}\left(R^{n-1}\right)\right)$ can be identified in the algebraic and topological senses to the completion of $C_{c}^{\infty}\left(R^{n-1}\right)$ with respect to the norm

$$
\int_{R^{n-1}}\left|\xi^{\prime}\right|^{2(m-j-1 / 2)(1-\theta)}\left(1+\left|\xi^{\prime}\right|\right)^{-2(j+1 / 2) \theta}\left|\hat{\phi}\left(\xi^{\prime}\right)\right|^{2} d \xi^{\prime},
$$

where $\hat{\phi}$ denotes the Fourier transform of $\phi$ and $\theta=1 / 2+\alpha \in(0,1)$.

\section{BIBLIOGRAPHY}

1. L. Hörmander and J.-L. Lions, Sur la complétion par rapport d une intégrale de Dirichlet, Math. Scand. 4 (1956), 259-270.

2. P. D. Lax and A. N. Milgram, Parabolic equations, Annals of Mathematics Studies, No. 33, Princeton Univ. Press, Princeton, N. J., 1954; pp. 167-190. 177.

3. J.-L. Lions, Sur les espaces d'interpolation; dualité, Math. Scand. 9 (1961), 147-

4. J.-L. Lions and E. Magenes, Problémes aux limites non homogènes. I, III, IV, V, Ann. Scuola Norm. Sup. Pisa 14 (1960), 259-308; ibid. 15 (1961), 39-101; ibid. 15 (1961), 311-326; ibid. 16 (1962), 1-44.

5. - Problèmes aux limites non homogènes. II, Ann. Inst. Fourier (Grenoble) 11 (1961), 137-178.

6. S. L. Sobolev, Some applications of functional analysis in mathematical physics, Izdat. Leningrad Gos. Univ., Leningrad, 1950.

BRANDEIS UNIVERSITY AND

Faculdade de Ciencias Economicas, Sao Paulo, Brazil 